\title{
Quasi-trapped ion and electron populations at Mercury
}

\author{
David Schriver, ${ }^{1,2}$ Pavel M. Trávníček, ${ }^{3,4}$ Brian J. Anderson, ${ }^{5}$ Maha Ashour-Abdalla, ${ }^{1,2}$ \\ Daniel N. Baker, ${ }^{6}$ Mehda Benna, ${ }^{7}$ Scott A. Boardsen, ${ }^{8}$ Robert E. Gold, ${ }^{5}$ Petr Hellinger, ${ }^{4}$ \\ George C. Ho, ${ }^{5}$ Haje Korth, ${ }^{5}$ Stamatios M. Krimigis, ${ }^{5,9}$ Ralph L. McNutt Jr., ${ }^{5}$ \\ Jim M. Raines, ${ }^{10}$ Robert L. Richard, ${ }^{1,2}$ James A. Slavin, ${ }^{10}$ Sean C. Solomon, ${ }^{11}$ \\ Richard D. Starr, ${ }^{12}$ and Thomas H. Zurbuchen ${ }^{10}$
}

Received 9 September 2011; revised 28 October 2011; accepted 31 October 2011; published 14 December 2011.

[1] Mariner 10 and MESSENGER spacecraft observations have established that Mercury has an intrinsic magnetic field and magnetosphere. Following the March 2011 insertion of MESSENGER into orbit around Mercury, measurements show that ions and electrons with typical energies of about $1-10 \mathrm{keV}$ form an equatorially centered distribution of plasma at $1.4 R_{\mathrm{M}}$ radial distance (where $R_{\mathrm{M}}$ is Mercury's radius) around a substantial portion of the planet in local time from morning through night and into the afternoon sector. Coincident with the detection of plasma around Mercury, an observed drop in the total magnetic pressure is attributable to the ion and electron thermal pressure. Additionally, intense waves near the ion cyclotron frequency were observed at the same location as the quasi-trapped particle population, which are likely a result of anisotropic distributions created by the large loss cone $\left(>30^{\circ}\right)$ at these radial distances. Citation: Schriver, D., et al. (2011), Quasi-trapped ion and electron populations at Mercury, Geophys. Res. Lett., 38, L23103, doi:10.1029/2011GL049629.

\section{Introduction}

[2] Mercury, the closest planet to the Sun, has the distinction of having the smallest planetary magnetosphere in our solar system [Russell et al., 1988]. Mariner 10 data from

\footnotetext{
${ }^{1}$ Institute of Geophysics and Planetary Physics, University of California, Los Angeles, California, USA.

${ }^{2}$ Department of Physics and Astronomy, University of California, Los Angeles, California, USA.

${ }^{3}$ Space Sciences Laboratory, University of California, Berkeley, California, USA.

${ }^{4}$ Astronomical Institute and Institute of Atmospheric Physics, Academy of Sciences of the Czech Republic, Prague, Czech Republic.

${ }^{5}$ Johns Hopkins University Applied Physics Laboratory, Laurel, Maryland, USA

${ }^{6}$ Laboratory for Atmospheric and Space Physics, University of Colorado at Boulder, Boulder, Colorado, USA.

${ }^{7}$ Solar System Exploration Division, NASA Goddard Space Flight Center, Greenbelt, Maryland, USA.

${ }^{8}$ Heliophysics Science Division, NASA Goddard Space Flight Center, Greenbelt, Maryland, USA.

${ }^{9}$ Office of Space Research and Technology, Academy of Athens, Athens, Greece.

${ }^{10}$ Department of Atmospheric, Oceanic and Space Sciences, University of Michigan, Ann Arbor, Michigan, USA.

${ }^{11}$ Department of Terrestrial Magnetism, Carnegie Institution of Washington, Washington, D. C., USA.

${ }^{12}$ Physics Department, Catholic University of America, Washington, D. C., USA.
}

Copyright 2011 by the American Geophysical Union. 0094-8276/11/2011GL049629 the 1970s established that Mercury has an intrinsic magnetic field, and this inference has been confirmed by magnetic field observations by the MErcury Surface, Space ENvironment, GEochemistry, and Ranging (MESSENGER) spacecraft. The planet has a dipole moment of $\sim 195 \mathrm{nT} R_{\mathrm{M}}^{3}$ (where $R_{\mathrm{M}}$ is Mercury's radius, or $2440 \mathrm{~km}$ ) and a tilt with respect to the planetary rotation axis of no more than $5^{\circ}$ [Ness et al., 1974; Jackson and Beard, 1977; Anderson et al., 2008]. Since the insertion of the MESSENGER spacecraft into orbit around Mercury in March 2011, Magnetometer data have confirmed these properties and also have shown that the intrinsic dipole magnetic field has an offset of 450 to $500 \mathrm{~km}$ north of the geographic equator [Anderson et al., 2011].

[3] Since the discovery of Mercury's intrinsic magnetic field and associated magnetosphere, it has been debated whether Mercury could have an equatorially trapped particle population analogous to the Earth's radiation belts or ring current [e.g., Baker et al., 1987]. Mercury has a much smaller magnetic dipole moment than Earth and given a scaling factor of 8 between the two magnetospheres based on their respective magnetopause locations, it is estimated that an Earth-like radiation belt, located at about 4-8 $R_{\mathrm{E}}$ ( $R_{\mathrm{E}}$ is Earth's radius, or $6371 \mathrm{~km}$ ) radial distance at Earth would be located at $<1 R_{\mathrm{M}}$, precluding the existence of a trapped particle population around Mercury. Recently, however, global three-dimensional hybrid simulations of a Mercury-like magnetosphere have shown that for relatively low solar wind pressure a quasi-trapped equatorial proton population could exist at radial distances between $\sim 1.3$ and $1.5 R_{\mathrm{M}}$ just inside the dayside magnetopause [Trávníček et al., 2009, 2010]. Also, numerical calculations of sodium ions in a global model of Mercury have shown that a sodium ion ring is possible [Yagi et al., 2010].

[4] The MESSENGER flybys of Mercury in 2008 and 2009 hinted at the existence of an equatorial plasma population around the planet when, prior to and after closest approach in the nightside equatorial plane near midnight at a radial distance $\sim 1.4 R_{\mathrm{M}}$, a decrease in the total magnetic field of $\sim 20$ to $40 \mathrm{nT}$ occurred at the same time that ions and electrons of moderate energy $(\sim \mathrm{keV})$ were observed [Slavin et al., 2008, 2009; Zurbuchen et al., 2008]. MESSENGER's present orbit around Mercury provides nearly continuous data coverage through the equatorial region around the planet. Observations show that for a majority of the passes through the equator at local times varying from morning through midnight and around to the afternoon, a moderately energetic $(\sim 1-10 \mathrm{keV})$ plasma population and associated diamagnetic decrease in the total magnetic field were evident 


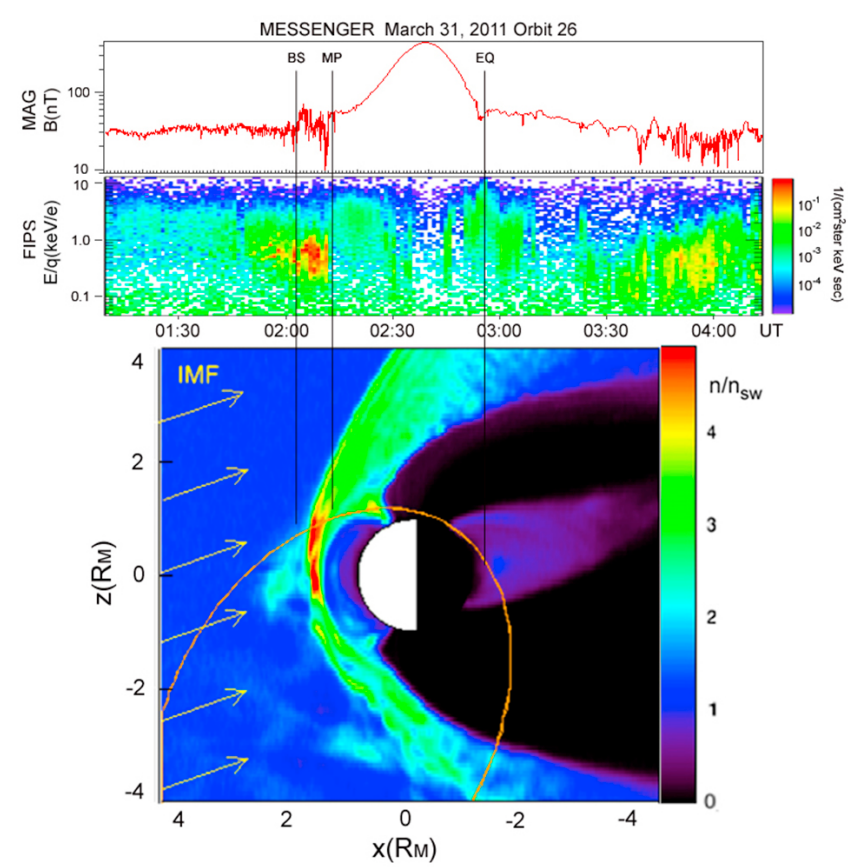

Figure 1. The first and second panels show about 3 hours of data from MAG and FIPS on the MESSENGER spacecraft during Mercury orbit 26 on March 31, 2011, respectively, and the third panel shows contours of ion density (normalized to solar wind density) from a global hybrid simulation in the noon-midnight meridian $(z-x$ plane, $y=0)$. Superimposed on the simulation contours is the MESSENGER orbit in gold; the arrows at left show the orientation of the IMF. The bow shock (BS), magnetopause (MP), and geographic equatorial (EQ) locations are indicated.

[Korth et al., 2011], as were intense electromagnetic ion cyclotron (EMIC) waves. These MESSENGER observations support the conclusions from simulations that a quasitrapped equatorial particle population is present around Mercury during relatively quiet solar conditions with low solar wind dynamic pressure.

[5] This paper addresses the question of the existence of a quasi-trapped particle population at Mercury. The outline of the paper is as follows. Spacecraft data and a simulation model are discussed in section 2 , results are presented in section 3, and conclusions are given in section 4 .

\section{Spacecraft Data and Simulation Model}

[6] This study uses magnetic field data from MESSENGER's fluxgate Magnetometer (MAG) [Anderson et al., 2007] and plasma data from the Energetic Particle and Plasma Spectrometer (EPPS) [Andrews et al., 2007]. EPPS has two detector systems. One is the Fast Imaging Plasma Spectrometer (FIPS) that measures ions with energy-tocharge ratios of 45-13,500 eV/e, and the other is the Energetic Particle Spectrometer (EPS), which measures ions with $15 \mathrm{keV}$ to $3 \mathrm{MeV}$ and electrons with $35 \mathrm{keV}$ to $1 \mathrm{MeV}$. The X-Ray Spectrometer (XRS) is used as a proxy to detect $\sim 1-10 \mathrm{keV}$ electrons [Slavin et al., 2008; Ho et al., 2011], below the EPS energy cutoff.

[7] MESSENGER was inserted into an eccentric nearpolar orbit around Mercury on March 18, 2011, at about 00:45 UTC. The orbit has approximately a 12 hour period, is inclined $82.5^{\circ}$ to Mercury's geographic equator, and had an initial periapsis at $200 \mathrm{~km}$ altitude and $60^{\circ} \mathrm{N}$ latitude and $15,193 \mathrm{~km}$ apoapsis altitude. MESSENGER crosses the geographic equator just after periapsis at a radial distance of $\sim 1.4 R_{\mathrm{M}}$; the crossing shortly after orbit insertion was at about $3.5 \mathrm{hr}$ magnetic local time (MLT) in the pre-dawn, post-midnight sector. The change in orbital node longitude between orbits varied from $\sim 3^{\circ}$ initially to $<2^{\circ}$, and the radial distance at the equator slowly increased from $1.39 R_{\mathrm{M}}$ on March 24 (orbit 12) to $1.49 R_{\mathrm{M}}$ on May 18, 2011 (orbit 122).

[8] Global hybrid simulations, which follow the selfconsistent evolution of the system by treating the plasma as particle ions and massless electrons, are used to provide global context for the MESSENGER observations at Mercury. Results discussed here are from the runs described by Trávníček et al. [2009, 2010]. These hybrid simulation runs included a Mercury-sized magnetic dipole moment (equivalent to $250 \mathrm{nT} R_{\mathrm{M}}^{3}$ ) placed at the geographic center of the planet with no tilt, and a solar wind speed of $450 \mathrm{~km} / \mathrm{s}$ and density of $15 \mathrm{~cm}^{-3}$. The simulation run here had an interplanetary magnetic field (IMF) directed northward with a large radial IMF component directed away from the Sun. These solar wind conditions correspond approximately to quiet times near aphelion where the solar wind density and speed are lower than at perihelion.

\section{Simulation and Data Results}

[9] To place the MESSENGER observations in a global context during a single orbit, data from MAG and FIPS, along with hybrid simulation results, are presented in Figure 1, in the first, second, and third panels, respectively. Starting at 01:09 UTC MESSENGER was in the solar wind approaching Mercury from the south. Ion density enhancements detected by FIPS upstream of the bow shock, along with enhanced magnetic fluctuations seen by MAG at about 01:30 UTC, indicate that the spacecraft moved through the foreshock region, which in a manner similar to that at Earth is formed where some solar wind ions and electrons reflect off the quasi-parallel bow shock and travel sunward (upstream) parallel to the IMF [e.g., Asbridge et al., 1968]. MESSENGER then crossed the bow shock (BS) into the magnetosheath, a region of slower-moving thermalized solar wind plasma downstream of the bow shock, at about 02:03 UTC, as shown by an increase in the magnetic field and ion flux.

[10] The spacecraft crossed the magnetopause (MP) boundary, which demarcates the passage from the solar wind into Mercury's magnetosphere, at about 02:12 UTC, as shown by the abrupt drop in ion flux, an increase in the total magnetic field, and the sudden reduction in magnetic field fluctuations. As MESSENGER approached the planet, the overall strength of the magnetic field increased as seen by MAG, and a population of higher-energy ions was seen by FIPS at about 02:20 UTC, which corresponds to the dayside cusp region [Zurbuchen et al., 2011]. The spacecraft then passed over the north pole into the lobe region, which has very low plasma density and where magnetic field lines have one end connected to the planet and the other end open far downstream. After closest approach (02:40 UTC), when the magnetic field reached its maximum, MESSENGER then crossed the geographic equator (GEQ, or $z=0)$ on the nightside at about 02:56 UTC, where a noticeable 

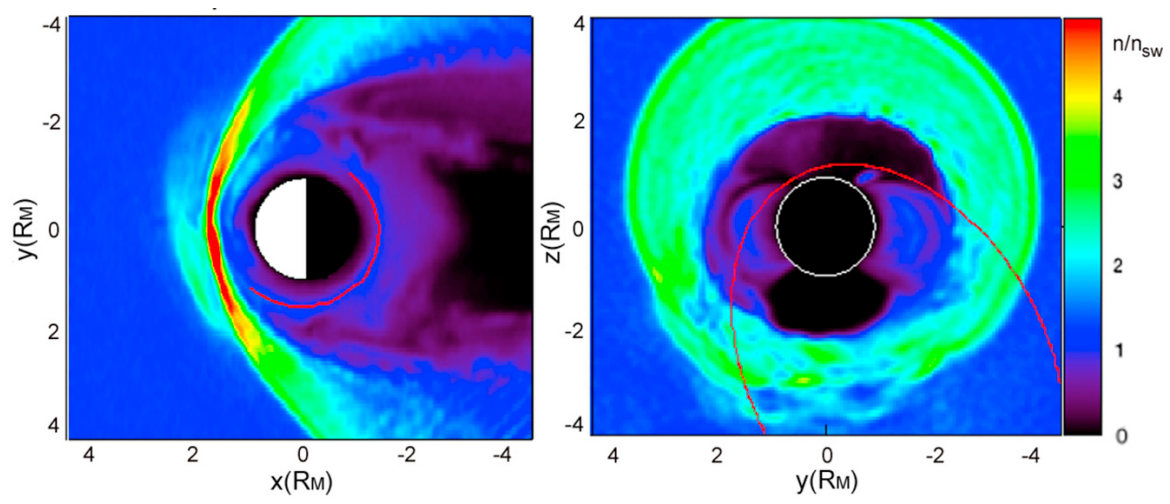

Figure 2. Color contours of ion density (normalized to the solar wind density) are shown in the geographic equatorial $(y-x)$ plane $(z=0)$ on the left and in the dusk-dawn meridian $(z-y)$ plane $(x=0)$ on the right. The equatorial plane is viewed from the north, with the Sun to the left, and the dusk-dawn meridian plane is viewed sunward from the night side. (left) The equatorial crossing positions of MESSENGER are shown superimposed in red from orbit 12 on March 24, 2011, to orbit 122 on May 18, 2011. (right) Orbit 92 on May 3, 2011, is superimposed in red.

depression of the total magnetic field of about $30 \mathrm{nT}$ was observed, coincident with the appearance of a relatively energetic $(\sim 5 \mathrm{keV})$ ion population. This location corresponds in the simulation to the region containing the equatorial quasi-trapped particle population. Then heading southbound, the spacecraft passed through the southern lobe, outbound through the magnetopause at about 03:50 UTC into the magnetosheath and eventually out into the solar wind again. Although the locations, extent, and detailed characteristics of the different regions and boundaries vary from orbit to orbit due to ever-changing solar wind conditions, the overall pattern of the magnetospheric structures seen in the orbital pass shown in Figure 1 was similar for the majority of orbits. In general there is very good correspondence between the simulations and observational data, although it must be kept in mind that this is only a highlevel comparison, with differences expected due to overall simulation limitations in terms of approximate system scaling to Mercury's dipole magnetic moment and offset (which is not taken into account) and the use of generic solar wind conditions that are an approximation of the actual solar wind conditions at the time of observation.

[11] The focus here is on the times when MESSENGER passed through the equatorial region just after periapsis, which occurred at $\sim 02: 56$ UTC in Figure 1. This is the region where a trapped particle population was found in the global kinetic simulations, as illustrated in Figure 2, which shows ion density contours (normalized to solar wind ion density) in the geographic equatorial plane (Figure 2, left) and in the dusk-dawn meridian plane (Figure 2, right). The red curve in Figure 2 (left) represents the location of all of the MESSENGER orbital passes through the geographic equator from March 24, 2011 (orbit 12), through May 18, 2011 (orbit 122). MESSENGER orbit 92 on May 3, 2011, when the spacecraft was near $x=0$, is shown in Figure 2 (right).

[12] As can be seen in Figure 2, the MESSENGER orbits during this time period were ideally suited to examine the equatorial particle distribution found in the simulations. In the nightside planetary magnetosphere, plasma-sheet particles convect anti-sunward toward the planet, as seen in the third panel of Figure 1. At the inner edge of the plasma sheet, close to the planet, the magnetic field becomes dipolar and plasma particles execute bounce motion along dipole field lines and drift around the planet azimuthally due to the gradient and curvature of the magnetic field $\mathbf{B}$, i.e., $\operatorname{grad} B$ and radius-of-curvature drifts [e.g., Northrop, 1963]. These azimuthal drifts are charge-dependent: ions drift clockwise around the planet as seen from the north and electrons counterclockwise. Near midnight the inner edge of the plasma sheet merges with the location of the quasi-trapped particle population and feeds into this population, acting as a plasma source from which the ions begin drifting toward dusk. As this drifting population moves around the planet to about $18 \mathrm{hr}$ MLT (dusk) it becomes distinct from the inner plasma sheet, as can be seen in Figure 2. For northward IMF conditions and low solar wind pressure the simulations show some ions can drift around the dayside to near noon and continue to make a complete circuit around the planet. Other ions are lost either by precipitation or by encountering the magnetopause on the dayside. For southward-directed IMF, a less complete plasma belt forms from post-dawn around the nightside to afternoon, with most particles hitting the magnetopause pre-noon before completing a full circuit around the planet [Trávníček et al., 2010].

[13] Data from equatorial crossings at different local times are shown in Figure 3. The chosen MLTs are post-midnight (first column), pre-midnight (second column), and very close to dusk (third column). The geographic equator and magnetic equator (MEQ) crossings are marked in each set of panels. As found by Anderson et al. [2011] from MAG data after MESSENGER orbit insertion, the magnetic equator is offset $484 \pm 11 \mathrm{~km}$ to the north of the geographic equator. Because MESSENGER passes over the north pole before heading south, it crosses the magnetic equator about 3 to 4 minutes before crossing the geographic equator. In each case, a magnetic depression can be seen close to the magnetic and geographic equatorial planes, with a total magnetic field decrease up to $40 \mathrm{nT}$ (first row), accompanied by the appearance of $\sim 5 \mathrm{keV}$ protons (second row), and for two of the events $1-10 \mathrm{keV}$ electrons, as indicated by the increase in counts from the XRS gas proportional counters (GPCs) in the third row [e.g., Slavin et al., 2008; Ho et al., 2011]. In the third event (orbit 90, third column of Figure 3), the XRS GPC levels hit saturation just prior to 07:30 UTC, triggering an automatic 5 min shutdown, which occurred before the 

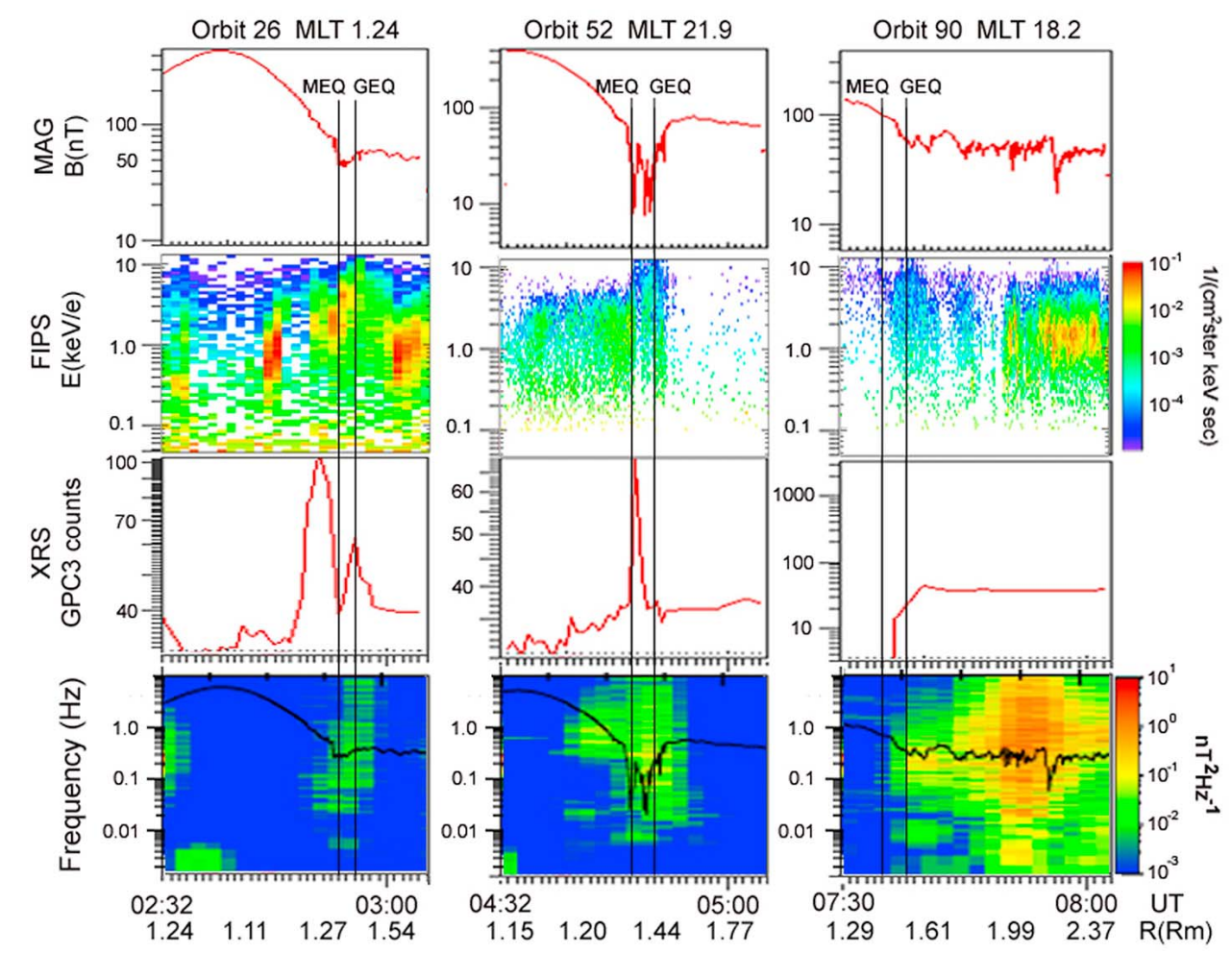

Figure 3. MAG (first row), FIPS (second row), XRS (third row) and magnetic field spectrogram (fourth row) data are shown for three different orbital crossings of the equator, orbit 26 on March 31, 2011 (first column), orbit 52 on April 13, 2011 (second column), and orbit 90 on May 2, 2011 (third column). The solid black curve in the wave spectrograms (fourth row) corresponds to the proton gyrofrequency. The times when MESSENGER crossed the geographic and magnetic equators are marked GEQ and MEQ, respectively.

spacecraft crossed the equator, so no XRS data are available for that time. These XRS saturation events were common at higher latitudes on the dusk side; however, their cause is beyond the scope of this paper. The magnetic wave spectrograms in the fourth row show strong wave activity centered on the proton gyrofrequency (shown by the solid black curve), coincident with the magnetic field depression and the presence of the 1-10 keV ions and electrons. Only the perpendicular component of the magnetic wave spectrum is shown in Figure 3, but the waves quite often have a parallel (compressional) component as well. The wave spectra are typically centered just below or above the proton gyrofrequency but vary somewhat from orbit to orbit, sometimes broadbanded as in Figure 3 but at other times narrowbanded. The emissions below the proton gyrofrequency may correspond to sodium ion cyclotron waves $\mathrm{CNa}^{+}$has $\mathrm{amu}=22.9$ ). Sodium ions, which are of planetary origin, have been observed by FIPS in the equatorial region on some passes [Zurbuchen et al., 2008, 2011].

[14] The data in Figure 3 show that four different indicators are observed almost simultaneously near the magnetic and geographic equators: (1) a magnetic depression of 5-50 nT, (2) ions with energies of $1-10 \mathrm{keV}$, (3) electrons with energies of $1-10 \mathrm{keV}$, and (4) magnetic wave oscillations near the proton gyrofrequency. A total of 102 equatorial crossings have been examined from orbit 12 on March 24, 2011, to orbit 115 on May 14, 2011. Out of all 102 equatorial crossings examined, 97 had one or more of the four indicators of a quasi-trapped particle population around Mercury. During some of the crossings FIPS and/or
XRS data were not available. MAG data were available on almost every crossing and provided the most reliable indicator in terms of the magnetic depression [Korth et al., 2011] and wave activity. Thus in $93 \%$ of the equatorial crossings for which data first became available, extending from the pre-dawn sector (3.7 $\mathrm{hr}$ MLT) through midnight (24 hr MLT) to late afternoon (15.8 hr MLT), evidence of a quasi-trapped plasma population was observed. There was a distinct decrease in the population in the pre-midnight to dusk sector, reflected in relatively shallow magnetic depressions $(\sim 5 \mathrm{nT})$ and lower proton energies $(\sim 1 \mathrm{keV})$ and fluxes. Nevertheless, only three crossings in that region showed no indication of a quasi-trapped population. Indeed many crossings showed relatively strong signals with magnetic depressions of $10 \mathrm{nT}$ or more and ions with $\sim 3-4 \mathrm{keV}$ energies [Korth et al., 2011]. The variation in the indicators is likely due to the changing solar wind conditions [e.g., Baker et al., 2011], i.e., if the solar wind pressure becomes larger then the magnetopause boundary pushes closer to the planet's dayside surface, reducing the possibility that ions and electrons can become trapped and drift around the planet.

[15] Data from EPS, which can detect electrons with energies $>36 \mathrm{keV}$, have also been examined for the equatorial crossings. In $42 \%$ of the equatorial crossings, electrons were detected in the $36 \mathrm{keV}$ and $63 \mathrm{keV}$ channels, with most of those events observed from pre-midnight to postmidnight. An analysis of the pitch angles for two of the events for which $>36 \mathrm{keV}$ electrons were observed showed that the particles had a large pitch angle near $90^{\circ}$, but at the 
equatorial crossings for those events the look angle was limited to large pitch angles, and it cannot be determined where the peak in pitch angle occurred, only that for those particles the pitch angles were large. The $1-10 \mathrm{keV}$ electrons (from XRS) were observed for about $80 \%$ of the cases, although in many of the cases without such electrons XRS was in a safe-mode when crossing the equator as a result of saturation of the GPC detector at higher latitudes. The observation of the higher-energy electrons $(>36 \mathrm{keV})$ is consistent with electrons that originate from the magnetotail plasma sheet, convect in close to the planet $\left(\sim 1.5 R_{\mathrm{M}}\right)$ near midnight, and then drift toward the dawn direction. Since there are almost no cases where $>36 \mathrm{keV}$ electrons are observed on the afternoon-dusk side, whereas $1-10 \mathrm{keV}$ electrons are often observed, it appears that the higherenergy electrons are lost in the dawn-morning sector, either through precipitation or by hitting the magnetopause, and do not make complete drift orbits around the planet.

[16] As Mercury has rotated beneath the MESSENGER orbit, the radial distance at the equatorial crossing has been slowly increasing. Starting with about orbit $115(15.8 \mathrm{hr}$ MLT), where the radial distance at the equator was $1.48 R_{\mathrm{M}}$, as MESSENGER progressed farther around the afternoon sector towards noon most of the equatorial crossings took place in the magnetosheath, i.e., beyond the magnetopause in the shock-thermalized solar wind, and there is no way in these cases to determine whether a quasi-trapped population was present inside the magnetosphere. The hybrid simulations (e.g., see Figure 2) indicate that the magnetopause nose location is at about $1.5 R_{\mathrm{M}}$ for low to moderate solar wind pressure, and that for northward IMF the quasi-trapped population is just planetward of this location.

\section{Conclusions}

[17] Since the MESSENGER spacecraft entered orbit around Mercury in March 2011, it has had a trajectory ideally suited to examine the plasma population in the equatorial region around the planet. Data from different instruments (MAG, EPPS, and XRS) have shown that a population of $1-10 \mathrm{keV}$ ions and electrons are present at a radial distance of $1.4-1.5 R_{\mathrm{M}}$. Somewhat higher-energy $(>36 \mathrm{keV})$ electrons were also observed, but these were confined primarily to the midnight, post-midnight, and pre-dawn sectors. The MESSENGER data for the MLT regions sampled around the planet thus far (morning to nightside through the afternoon) support the findings from previous simulations that Mercury has a quasi-trapped equatorially centered particle population for low to moderate solar wind pressures.

[18] Geomagnetically trapped particles in a dipole-type field configuration execute different types of motion [Hamlin et al., 1961] for which basic parameters can be determined at Mercury from the MESSENGER observations. One is the gyroradius around the magnetic field, which for a proton at the magnetic equator $(\sim 83 \mathrm{nT})$, with an average energy of $5 \mathrm{keV}$, is $\sim 87 \mathrm{~km}\left(0.036 R_{\mathrm{M}}\right)$. Thus a quasi-trapped proton at about $1.4 R_{\mathrm{M}}$ can gyrate about the magnetic field without striking the planet or the dayside magnetopause (located at about $1.5 R_{\mathrm{M}}$ ) as it drifts around the planet. The sodium ion gyroradius is $416 \mathrm{~km}\left(0.17 R_{\mathrm{M}}\right)$, so loss at the magnetopause on the dayside region becomes significant and trapping is less likely [e.g., Delcourt et al.,
2011], although lower-energy sodium ions could become trapped to form a ring around Mercury [Yagi et al., 2010].

[19] Another type of motion executed by a quasi-trapped particle is bounce motion along the dipolar magnetic field lines, which depends on the particle pitch angle. If the pitch angle is less than the loss-cone angle $\left(\theta_{\mathrm{LC}}\right)$, the particle will be lost and precipitate, otherwise the particle undergoes trapped mirror bounce motion along the magnetic field. From observed magnetic field values and dipole field line mapping to the surface of the planet, $\theta_{\mathrm{LC}} \sim 30^{\circ}$. The result of such a large loss cone is a "hole" in the velocity space distribution function that can be unstable to ion cyclotron waves [Cornwall et al., 1970], which can account for the strong EMIC wave activity observed by MESSENGER accompanying the quasi-trapped population.

[20] The term quasi-trapped is used to describe the particle population surrounding Mercury since global kinetic simulations indicate that only about $10 \%$ of the particles make a complete drift orbit around the planet, while the rest are lost by precipitating at the planet or by hitting the magnetopause. Despite the low percentage of fully trapped particles there is a constant feeding of plasma from different source regions on a timescale comparable to those for particle losses such that a particle population is continuously maintained around the planet. The MESSENGER observations indicate that the quasi-trapped ion population is stronger on the dawn side than the dusk side, which is the opposite of what is observed for Earth's ring current. The global hybrid simulations [Trávníček et al., 2010] and heavy ion trajectory calculations [Yagi et al., 2010] indicate that the dawn-dusk asymmetry at Mercury is due to the plasma sources for the quasi-trapped particle population at Mercury, which include the magnetotail plasma sheet, the low-latitude boundary layer (LLBL), and the planet itself. Convection in the magnetotail plasma sheet brings plasma toward the nightside of the planet and provides a variable but consistent source of hot $(\sim \mathrm{keV})$ particles near midnight. The LLBL provides plasma at the equatorial flanks, where magnetosheath plasma can enter the magnetosphere through Kelvin-Helmholtz instability and shear-related diffusion [Slavin et al., 2009; Trávnićek et al., 2009]. Mercury provides ions that come off its surface through a variety of processes, including photo-stimulated desorption, micrometeoroid bombardment, particle sputtering, and electron-stimulated desorption [Killen et al., 2004; Schriver et al., 2011]. The different sources combined are able to maintain a continuous quasi-trapped belt of particles around Mercury for low to moderate solar wind pressures, but in contrast to the situation at Earth, major contributions of plasma come into the dawn side from the LLBL and the planet [Trávnićek et al., 2010; Yagi et al., 2010].

[21] The quasi-trapped particle belt around Mercury in general has relatively low energies $(1-10 \mathrm{keV})$ and thus cannot be considered to be a radiation belt in the same sense as the trapped populations with $\mathrm{MeV}$ energies observed at Earth or the outer planets. At Mercury the quasi-trapped population would be more akin to Earth's ring current, at least energetically. One possible reason that the energies of a persistent quasi-trapped population at Mercury are lower is because for proton energies $\geq 50 \mathrm{keV}$, the corresponding particle gyroradius $(\geq 275 \mathrm{~km}$ ) would be too large to fit between the planet and the magnetopause. At Earth, the particle belt is much farther from the planet $\left(\sim 2-6 R_{\mathrm{E}}\right)$, and even very-high-energy particles can remain trapped for days, 
whereas the lifetime of a quasi-trapped particle at Mercury is on the order of a few minutes. Also because of the smaller magnetic field of Mercury, betatron acceleration that can occur as particles convect toward the planet [e.g., Kivelson et al., 1979] will not be as effective as at Earth due to a smaller ratio of the magnetic field between the magnetotail and inner magnetosphere. Additionally, wave-particle interactions that accelerate inner magnetospheric plasmas might be less effective at Mercury that at Earth.

[22] The results from the hybrid simulations and the MESSENGER orbital data analyzed thus far supporting the presence of a quasi-trapped particle population around Mercury imply that all of the planets in our solar system with internal magnetic fields host some sort of trapped region of charged particles. Compared to the other planets, Mercury's quasi-trapped particle population is closest to the surface. In general a trapped particle population around a planet with an internal field constitutes a persistent repository of energetic particles that can exert a major influence on the inner magnetosphere and have strong effects on the planetary surface via precipitation. At Mercury ion and electron precipitation fluxes due to the quasi-trapped population are substantial, $\sim 10^{9} \mathrm{~cm}^{-2} \mathrm{~s}^{-1}$ [Trávníček et al., 2010; Schriver et al., 2011], and can play a major role in exospheric formation and space weathering. This role and detailed properties of Mercury's quasi-trapped particle population will be illuminated as more MESSENGER data are returned.

[23] Acknowledgments. This work was supported by NASA MESSENGER grants NNX07AR62G, NNX07AV79G, and NNX09AD41G and contract ME09009 from the Czech Ministry of Education. Computing was carried out with NASA Advanced Supercomputing systems, NSF NCAR Frost Blue Gene, and the computing facility at the Institute of Atmospheric Physics, Prague, Czech Republic.

[24] The Editor thanks Wing Ip and Kanako Seki for their assistance in evaluating this paper.

\section{References}

Anderson, B. J., M. H. Acuña, D. A. Lohr, J. Scheifele, A. Raval, H. Korth, and J. A. Slavin (2007), The Magnetometer instrument on MESSENGER, Space Sci. Rev., 131, 417-450, doi:10.1007/s11214-007-9246-7.

Anderson, B. J., M. H. Acuña, H. Korth, M. E. Purucker, C. L. Johnson, J. A. Slavin, S. C. Solomon, and R. L. McNutt Jr. (2008), The structure of Mercury's magnetic field from MESSENGER's first flyby, Science, 321, 82-85, doi:10.1126/science. 1159081 .

Anderson, B. J., C. L. Johnson, H. Korth, M. E. Purucker, R. M. Winslow, J. A. Slavin, S. C. Solomon, R. L. McNutt Jr., J. M. Raines, and T. H. Zurbuchen (2011), The global magnetic field of Mercury from MESSENGER orbital observations, Science, 333, 1859-1862, doi:10.1126/ science. 1211001 .

Andrews, G. B., et al. (2007), The MESSENGER Energetic Particle and Plasma Spectrometer, Space Sci. Rev., 131, 523-556, doi:10.1007/ s11214-007-9272-5.

Asbridge, J., S. J. Bame, and I. B. Strong (1968), Outward flow of protons from the Earth's bow shock, J. Geophys. Res., 73, 5777-5782, doi:10.1029/JA073i017p05777.

Baker, D. N., J. E. Borovsky, J. O. Burns, G. R. Gisler, and M. Zeilik (1987), Possible calorimetric effects at Mercury due to solar windmagnetosphere interactions, J. Geophys. Res., 92, 4707-4712, doi:10.1029/ JA092iA05p04707.

Baker, D. N., et al. (2011), The space environment of Mercury at the times of the second and third MESSENGER flybys, Planet. Space Sci., 59, 2066-2074, doi:10.1016/j.pss.2011.01.018.

Cornwall, J. M., F. V. Coroniti, and R. M. Thorne (1970), Turbulent loss of ring current protons, J. Geophys. Res., 75, 4699-4709, doi:10.1029/ JA075i025p04699.

Delcourt, D. C., T. E. Moore, and M.-C. H. Fok (2011), On the effect of IMF turning on ion dynamics at Mercury, Ann. Geophys., 29, 987-996, doi:10.5194/angeo-29-987-2011.

Hamlin, D. A., R. Karplus, R. C. Vik, and K. M. Watson (1961), Mirror and azimuthal drift frequencies for geomagnetically trapped particles, J. Geophys. Res., 66, 1-4, doi:10.1029/JZ066i001p00001.
Ho, G. C., et al. (2011), Observations of suprathermal electrons in Mercury's magnetosphere during the three MESSENGER flybys, Planet. Space Sci., 59, 2016-2025, doi:10.1016/j.pss.2011.01.011.

Jackson, D. J., and D. B. Beard (1977), The magnetic field of Mercury, J. Geophys. Res., 82, 2828-2836, doi:10.1029/JA082i019p02828.

Killen, R. M., M. Sarantos, A. E. Potter, and P. Reiff (2004), Source rates and ion recycling rates for $\mathrm{Na}$ and $\mathrm{K}$ in Mercury's atmosphere, Icarus, 171, 1-19, doi:10.1016/j.icarus.2004.04.007.

Kivelson, M., S. M. Kaye, and D. J. Southwood (1979), The physics of plasma injection events, in Dynamics of the Magnetosphere, edited by S. I. Akasofu and D. Reidel, pp. 385-405, D. Reidel, Dordrecht, Netherlands.

Korth, H., B. J. Anderson, J. M. Raines, J. A. Slavin, T. H. Zurbuchen, C. L. Johnson, M. E. Purucker, R. M. Winslow, S. C. Solomon, and R. L. McNutt Jr. (2011), Plasma pressure in Mercury's equatorial magnetosphere derived from MESSENGER Magnetometer observations, Geophys. Res. Lett., 38, L22201, doi:10.1029/2011GL049451.

Ness, N. F., K. W. Behannon, R. P. Lepping, Y. C. Whang, and K. H. Schatten (1974), Magnetic field observations near Mercury: Preliminary results from Mariner 10, Science, 185, 151-160, doi:10.1126/science. 185.4146.151.

Northrop, T. G. (1963), The Adiabatic Motion of Charged Particles, 109 pp., Interscience, New York.

Russell, C. T., D. N. Baker, and J. A. Slavin (1988), The magnetosphere of Mercury, in Mercury, edited by F. Vilas, C. R. Chapman, and M. S. Matthews, pp. 514-561, Univ. of Ariz. Press, Tucson.

Schriver, D., et al. (2011), Electron transport and precipitation at Mercury during the MESSENGER flybys: Implications for electron-stimulated desorption, Planet. Space Sci., 59, 2026-2036, doi:10.1016/j. pss.2011.03.008

Slavin, J. A., et al. (2008), Mercury's magnetosphere after MESSENGER's first flyby, Science, 321, 85-89, doi:10.1126/science.1159040.

Slavin, J. A., et al. (2009), MESSENGER observations of magnetic reconnection in Mercury's magnetosphere, Science, 324, 606-610, doi:10.1126/science.1172011.

Trávníček, P. M., P. Hellinger, D. Schriver, D. Hercik, J. A. Slavin, and B. J. Anderson (2009), Kinetic instabilities in Mercury's magnetosphere Three-dimensional simulation results, Geophys. Res. Lett., 36, L07104, doi:10.1029/2008GL036630.

Trávníček, P. M., D. Schriver, P. Hellinger, D. Hercik, B. J. Anderson, M. Sarantos, and J. A. Slavin (2010), Mercury's magnetosphere-solar wind interaction for northward and southward interplanetary magnetic field: Hybrid simulation results, Icarus, 209, 11-22, doi:10.1016/j.icarus. 2010.01.008.

Yagi, M., K. Seki, Y. Matsumoto, D. C. Delcourt, and F. Leblanc (2010), Formation of a sodium ring in Mercury's magnetosphere, J. Geophys. Res., 115, A10253, doi:10.1029/2009JA015226.

Zurbuchen, T. H., J. M. Raines, G. Gloeckler, S. M. Krimigis, J. A. Slavin, P. L. Koehn, R. M. Killen, A. L. Sprague, R. L. McNutt Jr., and S. C. Solomon (2008), MESSENGER observations of the composition of Mercury's ionized exosphere and plasma environment, Science, 321, 90-92, doi:10.1126/science.1159314.

Zurbuchen, T. H., et al. (2011), MESSENGER observations of the spatial distribution of planetary ions near Mercury, Science, 333, 1862-1865, doi:10.1126/science.1211302.

B. J. Anderson, R. E. Gold, G. C. Ho, H. Korth, S. M. Krimigis, and R. L. McNutt Jr., Johns Hopkins University Applied Physics Laboratory, Laurel, MD 20723, USA.

M. Ashour-Abdalla, R. L. Richard, and D. Schriver, Institute of Geophysics and Planetary Physics, University of California, Los Angeles, CA 90095, USA. (dave@igpp.ucla.edu)

D. N. Baker, Laboratory for Atmospheric and Space Physics, University of Colorado at Boulder, Boulder, CO 80303, USA.

M. Benna, Solar System Exploration Division, NASA Goddard Space Flight Center, Greenbelt, MD 20771, USA.

S. A. Boardsen, Heliophysics Science Division, NASA Goddard Space Flight Center, Greenbelt, MD 20771, USA.

P. Hellinger, Astronomical Institute and Institute of Atmospheric Physics, AS CR, 14131 Prague, Czech Republic.

J. M. Raines, J. A. Slavin, and T. H. Zurbuchen, Department of Atmospheric, Oceanic and Space Sciences, University of Michigan, Ann Arbor, MI 48109, USA.

S. C. Solomon, Department of Terrestrial Magnetism, Carnegie Institution of Washington, Washington, DC 20015, USA.

R. D. Starr, Physics Department, Catholic University of America, Washington, DC 20064, USA.

P. M. Trávníček, Space Sciences Laboratory, University of California, Berkeley, CA 94720, USA. 